\title{
Determining the Distribution of Values of Stochastic Impulses Acting on a Discrete System in Relation to Their Intensity
}

\author{
M. JABŁOŃSKI ${ }^{a}$ AND A. OzGA ${ }^{b}$ \\ ${ }^{a}$ Faculty of Mathematics and Computer Science, Jagiellonian University \\ Gołębia 24, 31-007 Cracow, Poland \\ ${ }^{b}$ AGH University of Science and Technology, Faculty of Mechanical Engineering and Robotics \\ Department of Mechanics and Vibroacoustics, al. A. Mickiewicza 30, 30-059 Krakow, Poland
}

\begin{abstract}
In our previous works we introduced and applied a mathematical model that allowed us to calculate the approximate distribution of the values of stochastic impulses $\eta_{i}$ forcing vibrations of an oscillator with damping from the trajectory of its movement. The mathematical model describes correctly the functioning of a physical RLC system if the coefficient of damping is large and the intensity $\lambda$ of impulses is small. It is so because the inflow of energy is small and behaviour of RLC is stable. In this paper we are going to present some experiments which characterize the behaviour of an oscillator RLC in relation to the intensity parameter $\lambda$, precisely to $\lambda E(\eta)$. The parameter $\lambda$ is a constant in the exponential distribution of random variables $\tau_{i}$, where $\tau_{i}=t_{i}-t_{i-1}$, $i=1,2, \ldots$ are intervals between successive impulses.
\end{abstract}

PACS: $45.10 .-\mathrm{b}, 45.30 .+\mathrm{s}$

\section{Introduction}

In the paper we discuss the motion of an oscillator described by the equation

$$
\frac{\mathrm{d}^{2} x}{\mathrm{~d} t^{2}}+2 b \frac{\mathrm{d} x}{\mathrm{~d} t}+a^{2} x=f(t)
$$

with initial conditions

$$
x(0)=0 \text { and } \dot{x}(0)=0,
$$

where

$$
f(t)=\sum_{t_{i}<t} \eta_{i} \delta_{t_{i}}(t),
$$

$\eta_{i}$ is any sequence of real numbers, $t_{i}$ is any increasing sequence of real numbers, $\delta_{t_{i}}(t)$ are the Dirac distributions at $t_{i}\left(\delta_{t_{i}}(t)=\delta\left(t-t_{i}\right)\right)$ and the sum is taken over all $t_{i}$ such that $t_{i}<t$. We assume that $a>b>0$.

If $t_{i}$ and $\eta_{i}$ are random variables then the solution of Eqs. (1)-(3) is a stochastic process [1, 2] and it is given by the following formula:

$$
\begin{aligned}
& x(t)=\frac{1}{\sqrt{a^{2}-b^{2}}} \sum_{0<t_{i}<t} \eta_{i} \exp \left(-b\left(t-t_{i}\right)\right) \\
& \quad \times \sin \left(\sqrt{a^{2}-b^{2}}\left(t-t_{i}\right)\right) .
\end{aligned}
$$

If random variables $\eta_{i}$ and $\tau_{i}=t_{i}-t_{i-1}, i \in Z$, are stochastically independent, $\eta_{i}$ are identically distributed with finite expectation and $\tau_{i}=t_{i}-t_{i-1}$ with $\lim _{i \rightarrow-\infty} t_{i}=-\infty$ are also identically distributed with exponential distribution, then the process (the sum is taken over an infinite number of $t_{i}$ )

$$
x(t)=\frac{1}{\sqrt{a^{2}-b^{2}}} \sum_{t_{i}<t} \eta_{i} \exp \left(-b\left(t-t_{i}\right)\right)
$$

$$
\times \sin \left(\sqrt{a^{2}-b^{2}}\left(t-t_{i}\right)\right)
$$

is already stationary and is close to (4) for large $t$. The process (5) is also ergodic.

To determine theoretical stochastic moments $m_{n+1}$ of the process $x(t)$ given by (5), for $n \geq 0$ we can use the following equations (see $[3,4]$ )

$$
\begin{aligned}
& m_{n+1}=\sum_{j=0}^{n}\left(\begin{array}{l}
n \\
j
\end{array}\right) m_{(n-j)} \frac{\lambda E\left(\eta^{(j+1)}\right)}{c^{2+j}} C(j+1), \\
& n=0,1,2, \ldots
\end{aligned}
$$

where $c=\sqrt{a^{2}-b^{2}}$

$$
C(j)=\frac{j !}{\prod_{r=0}^{j / 2-1}\left[(j b / c)^{2}+(2 r)^{2}\right]} \frac{c}{j b}
$$

for $j$ even and $j>0$ and

$$
C(j)=\frac{j !}{\prod_{r=0}^{(j-1) / 2-1}\left[(j b / c)^{2}+(2 r+1)^{2}\right]}
$$

for $j$ odd and $j>0$.

If $\eta$ assumes a finite number of values $\left\{\eta_{1}, \eta_{2} \ldots \eta_{k}\right\}$ with probabilities $p_{i}=P\left(\eta=\eta_{i}\right)$ then, by (6), for any $n>0$ we get

$$
\begin{aligned}
& \sum_{i=1}^{k} p_{i}\left[\left(m_{n} m_{1}-m_{n+1}\right) \eta_{i}\right. \\
& \left.\quad+\sum_{j=1}^{n}\left(\begin{array}{c}
n \\
j
\end{array}\right) m_{(n-j)} m_{1} \eta_{i}^{(j+1)} \frac{C(j+1)}{C(1) c^{j}}\right]=0 .
\end{aligned}
$$

Equation (9) allows us to determine the distributions of impulses which forced the vibrations of an oscillator if 
$m_{i}$ is known. In practical applications we can find these numbers using the following formula:

$$
E\left(x^{n}(t)\right) \cong \frac{1}{k} \sum_{i=1}^{k} x^{n}(t i / k)
$$

for large $t$ and $k$. It is a consequence of stationarity and ergodicity.

The first partial mathematical results regarding vibration of oscillators forced by stochastic impulses can be found in works [5-11]. In all these studies the movement of an oscillator is described by linear equations, which gives merely an approximate description of a physical phenomenon. It turns out, for example, that the behavior of a real RLC system depends on the intensity of impulses and thus there are discrepancies between this system and the mathematical model. In the present work we discuss this problem. The aim of this paper is to present the results of some experiments which characterize the behavior of an oscillator RLC in relation to the intensity parameter $\lambda$, precisely to $\lambda E(\eta)$.

\section{The influence of intensity on the parameters $a$ and $b$ of an oscillator}

In order to test the possible practical applications of the theoretical considerations, electromechanical analogies were used and in the experiment an RCL system with capacity $C=2 \mathrm{nF}$ and inductivity $L=5 \mathrm{mH}$ that was applied. The forcing signal $\eta$ of the value equal to $10 \mathrm{~V}$ was generated on the analogue output of the card NI USB-6251 at the sampling rate of $1 \mathrm{MHz}$, with simultaneous recording of the system's response on the analogue input. Stochastic moments of excitation of the movement of the oscillator $t_{i}$ were generated in accordance with the mathematical assumptions presented above. The application was built in the Labview environment. The impulses were executed with the help of single samples of the shortest executable duration of $2 \times 10^{-6} \mathrm{~s}$, issuing from the sampling rate.

An analysis of the recorded response of a physical system to the stochastic impulses forcing the vibrations of an oscillator shows that for each impulse the parameters $c, b$, and $\eta$ depend on the duration of work of the oscillator (with the passage of time the physical system is getting warmer), the intensity of the impulses as well as the temperature of the environment. Our goal is to assess in what way these changes influence the differences between the stochastic moment computed in our mathematical model and the moment determined on the basis of the course of vibrations of an oscillator. Selected values of these parameters for the impulses represented in Fig. 1, taken at the 2nd second of the trajectory of oscillator's motion for the intensity $\lambda=200$ can be found in Table I.

The parameters of the response of the system, at which the theoretical system is close to the physical one can be determined on the basis of the stochastic moments calculated from the trajectory of the motion of the oscillator.

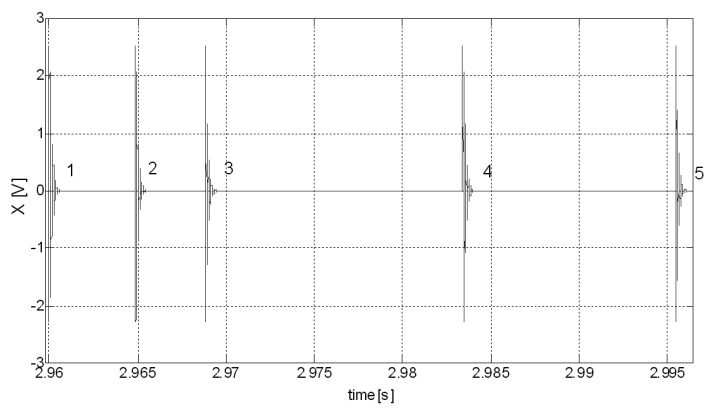

Fig. 1. The trajectory of vibrations of an oscillator RLC forced by impulses of the intensity $\lambda=200$.

TABLE I

Parameters $b, c$, and $\eta$ recorded at the fragment of the trajectory shown in Fig. 1.

\begin{tabular}{c|c|c|c}
\hline \hline The impulse & $b$ & $c$ & $\eta$ \\
\hline 1 & 8540.92 & 281940.77 & 742356.06 \\
2 & 8540.03 & 281942.46 & 743105.64 \\
3 & 8543.87 & 281944.60 & 742407.87 \\
4 & 8538.70 & 281942.63 & 742143.68 \\
5 & 8540.36 & 281943.91 & 742362.94
\end{tabular}

At the same time such a trajectory is perturbed by noise. The mean value of the noise $(-0.00014 \mathrm{~V})$ has a significant influence of the received results. An additional difficulty lies in charging of the capacitor during the impulse, which is marked with a rectangle in Fig. 2.

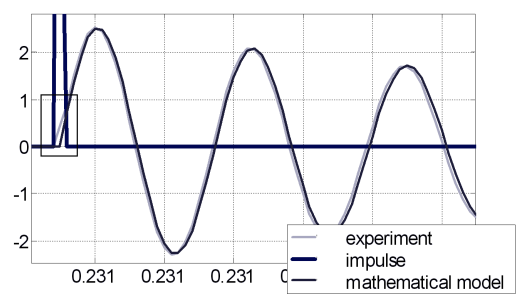

Fig. 2. Comparison of a mathematical model with the actual course on the basis of one impulse.

The first measurement sample of each of the impulses introduces erroneous data to the approximation of the parameter $c$ and to all three stochastic moments; moreover, the first of them is charged with the greatest error. Using in our computations the first sample of the system's response to the forcing impulse for the intensity $\lambda=100$ for $t=59.98 \mathrm{~s}$ we receive $m_{1}=0.00092740$, $m_{2}=0.01993046$, and $m_{3}=0.00426989$, rejecting this sample completely we receive $m_{1}=0.00088955, m_{2}=$ 0.01991461 , and $m_{3}=0.00426325$.

Another difficulty consists in the limitations of the algorithm connected with sampling and the duration of measurement. If the randomly selected distance between 
the impulses is smaller than $2 \times 10^{-6} \mathrm{~s}$, the algorithm which is responsible for generation of impulses ignores such an impulse. Moreover, the mathematical model is calculated for the time approaching infinity while we analyse the trajectory of the movement of an oscillator during one minute. One should not assume that for such a time interval the intensity has reached the demanded value. In order to determine the distributions of impulses it is necessary to adhere to the precision of $10^{-5} \mathrm{~V}$, which is connected with the necessity of application of algorithms determining the intensity that actually was achieved at the moment when the signal was recorded. And so, it was determined that for the imposed intensity $\lambda\{100,200,500\}$ for the calculations aimed at computation of parameters we should assume successively $\{98.209,197.050,498.615\}$.

Knowing these imperfections of the measurements and reducing their influence on the analysis of the recorded process we are able to select the parameters $c, b$, and $\eta$ of a theoretical oscillator so that the mathematical model with these parameters can best reflect an actual physical system working in given conditions. The determined stochastic moments from the recorded course and from the mathematical model for all intensities can be found in Figs. 3-8.

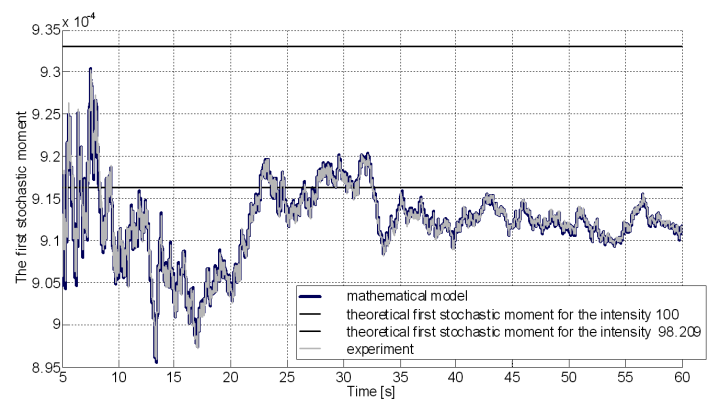

Fig. 3. The first stochastic moment determined from the experiment and from the mathematical model for $\lambda=100$.

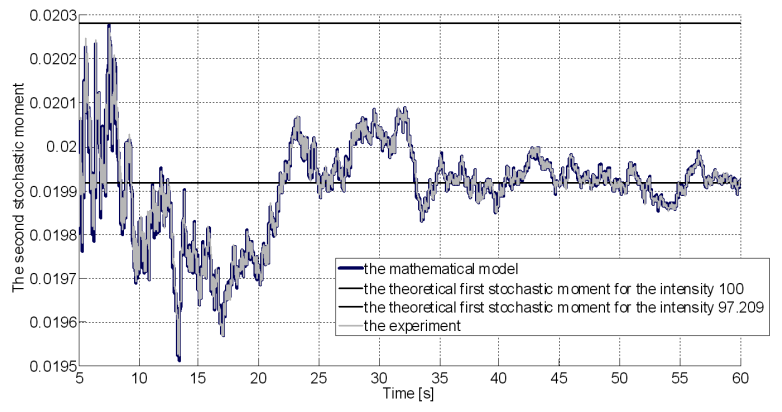

Fig. 4. The second stochastic moment determined from the experiment and from the mathematical model for $\lambda=100$.

The values of the parameters of the system's responses to the stochastic impulses calculated from the stochas-

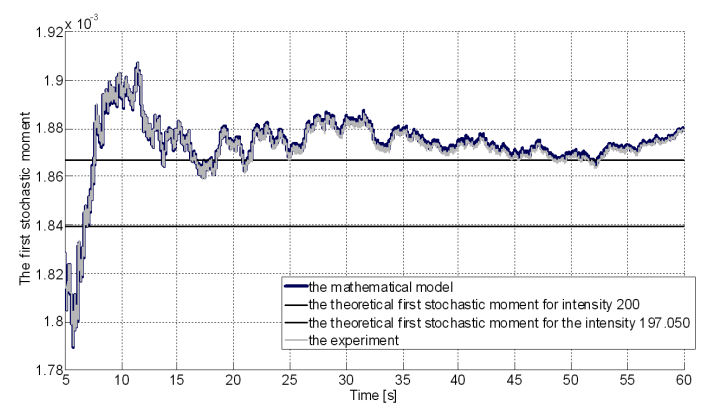

Fig. 5. The first stochastic moment determined from the experiment and from the mathematical model for $\lambda=200$.

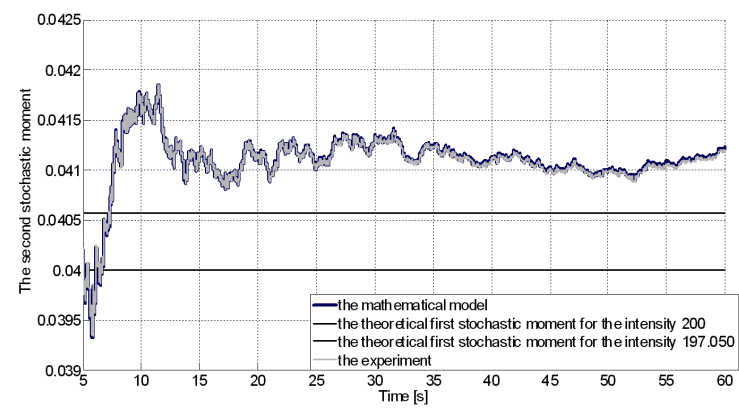

Fig. 6. The second stochastic moment determined from the experiment and from the mathematical model for $\lambda=200$.

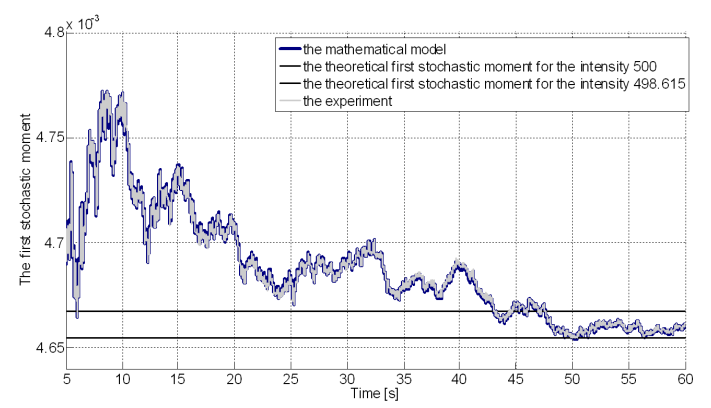

Fig. 7. The first stochastic moment determined from the experiment and from the mathematical model for $\lambda=500$.

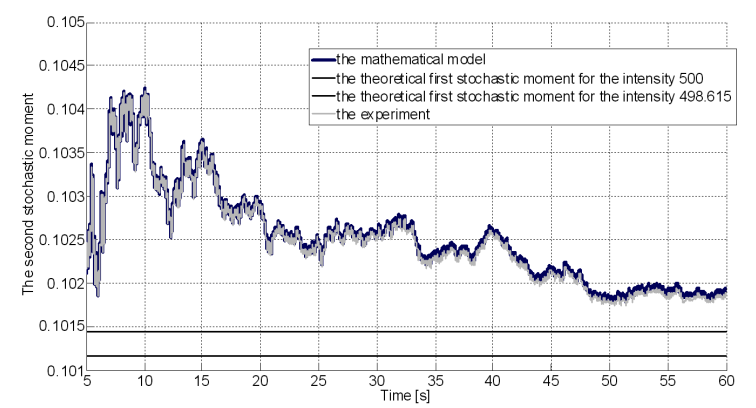

Fig. 8. The second stochastic moment determined from the experiment and from the mathematical model for $\lambda=500$. 
tic moments for all considered intensities can be found in Table II. It is worth mentioning that the sample with the intensity of 100 was recorded first; the remaining two samples were recorded after a two minute break between the measurements, hence the differences between the determined values of the parameters.

TABLE II

The values of the parameters of the system's response, calculated from the stochastic moments.

\begin{tabular}{c|c|c|c}
\hline \hline $\begin{array}{c}\text { The imposed } \\
\text { intensity } \lambda\end{array}$ & $b$ & $c$ & $\eta$ \\
\hline 100 & 8555 & 282221 & 743800 \\
200 & 8543 & 281940 & 742590 \\
500 & 8541 & 281862 & 742300
\end{tabular}

The same system RCL responds with different values of the parameters $b, c$, and $\eta$ for different intensities. The interpretation of the statistical data acquired in the experiments must take this into account.

\section{The influence of intensity on the time of the measurement}

It must be mentioned that it is important to select the duration of the experiment so that the stochastic moments computed from the trajectory of movement can provide the possibly most precise information about distributions. On the one hand, the time cannot be too long in order to prevent any significant change of the parameters of the system's response, and, on the other hand, it cannot be too short because it is necessary to calculate the stochastic moments with adequate precision.

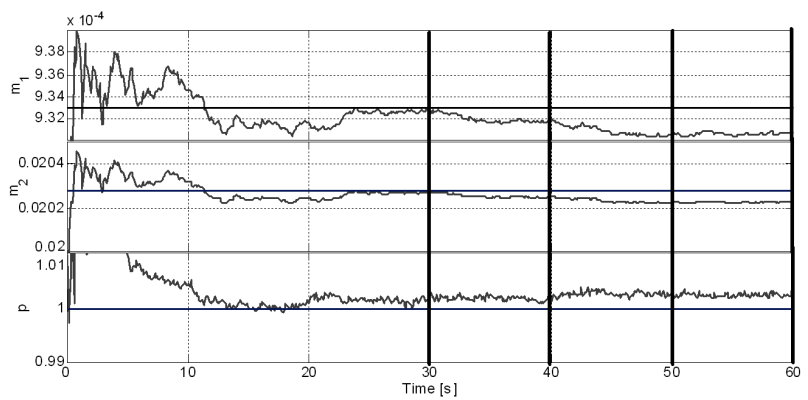

Fig. 9. The first and second stochastic moments calculated as the mean of 100 trajectories and the distribution of impulses for $\lambda=100$ determined on their basis.

Using the mathematical model, a simulation was performed of hundred trajectories of movement for each of the considered intensities, and for each trajectory the first two stochastic moments $m_{1}$ and $m_{2}$ were computed. With the help of the means of the moments, distributions of impulses were calculated from (9). In the diagrams

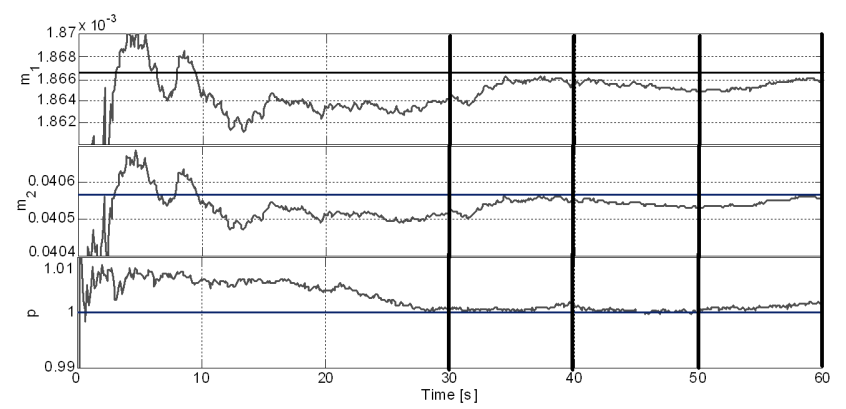

Fig. 10. As in Fig. 9, but for $\lambda=200$.

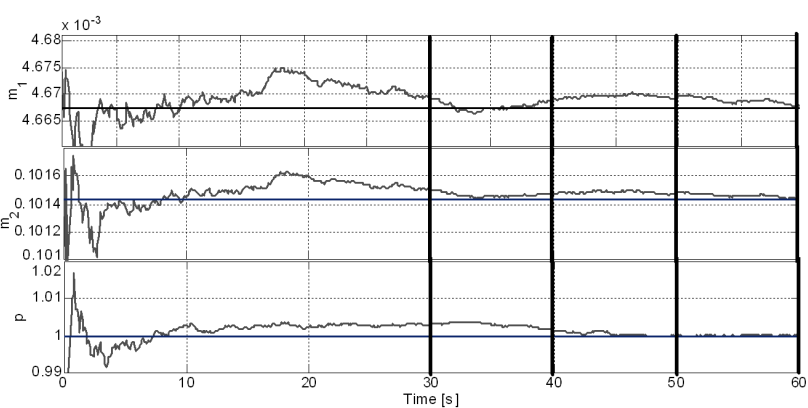

Fig. 11. As in Fig. 9, but for $\lambda=500$.

below we can see that for $\lambda=100$ the distribution of impulses may achieve the required value after $12 \mathrm{~s}$ (Fig. 9), for $\lambda=200$ after $30 \mathrm{~s}$ (Fig. 10) and for $\lambda=500$ after $42 \mathrm{~s}$ (Fig. 11).

Standard deviation calculated at 30, 40, 50, and $60 \mathrm{~s}$ for both stochastic moments $m_{1}$ and $m_{2}$ is shown in Table III. The longer the measurement time, the more precise results are obtained. With the growth of intensity, the standard deviation increases. The standard deviation for $\lambda=100$ after $30 \mathrm{~s}$ equals the standard deviation for $\lambda=200$ after $50 \mathrm{~s}$.

\section{Conclusions}

The intensity parameter $\lambda$, which characterizes the distribution of random intervals $\tau_{i}=t_{i}-t_{i-1}, i=1,2, \ldots$ between successive impulses, is the basic parameter influencing the behavior of an oscillating system. An analysis of experiments indicates that small perturbations of $\lambda$ change the stochastic moments of the process $x(t)$.

However, with a certain approximation, on the basis of the motion of the system we can determine the distributions of impulses. Additionally, the analysis carried out in our study indicates that:

- The parameters of the responses of oscillating systems forced by stochastic impulses do not change in a certain time so that an analysis of the system with the help of the mathematical model described in the introduction is possible. In our further research we are looking for mechanical systems as stable as it is possible. 
TABLE III

The values of parameters of the system's response calculated from the stochastic moments.

\begin{tabular}{c|c|c|c|c|c|c}
\hline \hline \multirow{2}{*}{$\begin{array}{c}\text { Time } \\
{[\mathrm{s}]}\end{array}$} & \multicolumn{2}{|c|}{$\lambda=100$} & \multicolumn{2}{c|}{$\lambda=200$} & \multicolumn{2}{c}{$\lambda=500$} \\
\cline { 2 - 7 } & $\delta m_{1}$ & $\delta m_{2}$ & $\delta m_{1}$ & $\delta m_{2}$ & $\delta m_{1}$ & $\delta m_{2}$ \\
\hline 30 & $1.785 \times 10^{-6}$ & $3.918 \times 10^{-5}$ & $2.129 \times 10^{-6}$ & $4.704 \times 10^{-5}$ & $3.863 \times 10^{-6}$ & $8.663 \times 10^{-5}$ \\
40 & $1.497 \times 10^{-6}$ & $3.278 \times 10^{-5}$ & $1.841 \times 10^{-6}$ & $4.009 \times 10^{-5}$ & $3.027 \times 10^{-6}$ & $6.812 \times 10^{-5}$ \\
50 & $1.361 \times 10^{-6}$ & $2.995 \times 10^{-5}$ & $1.650 \times 10^{-6}$ & $3.646 \times 10^{-5}$ & $2.823 \times 10^{-6}$ & $6.323 \times 10^{-5}$ \\
60 & $1.222 \times 10^{-6}$ & $2.674 \times 10^{-5}$ & $1.520 \times 10^{-6}$ & $3.398 \times 10^{-5}$ & $2.584 \times 10^{-6}$ & $5.878 \times 10^{-5}$
\end{tabular}

- The second stochastic moment is more susceptible to the above mentioned changes of parameters.

- The lesser the intensity, the greater is the impact of its perturbation on stochastic moments.

- The greater the intensity, the longer the trajectory of motion should be chosen for an analysis.

Vibrations of the systems forced by stochastic impulses are a complex process. In technological applications it will be necessary to obtain information coming at the same time from more than one recording device in the same measurement conditions and at the same working time for each device. Research whose results could be used for construction of an apparatus for continuous measurement of dust granulation still requires numerous experiments.

\section{Acknowledgments}

This work has been financed by the Polish Ministry of Science and Higher Education, research project No. N N501 180636.

\section{References}

[1] M. Jabłoński, A. Ozga, Mechanics 25, 156 (2006).

[2] M. Jabłonski, A. Ozga, Arch. Acoust. 34, 601 (2009).

[3] M. Jabłoński, A. Ozga, Acta Phys. Pol. A 118, 74 (2010).

[4] M. Jabłoński, A. Ozga, T. Korbiel, P. Pawlik, Acta Phys. Pol. A 119, 977 (2011).

[5] S.O. Rice, Bell System Techn. J. 23, 1 (1944).

[6] J.B. Roberts, J. Sound Vibrat. 2, 336 (1965).

[7] J.B. Roberts, J. Sound Vibrat. 2, 375 (1965).

[8] J.B. Roberts, J. Sound Vibrat. 24, 23 (1972).

[9] J.B. Roberts, J. Sound Vibrat. 28, 93 (1973).

[10] J.B. Roberts, P.D. Spanos, Int. J. Non-Linear Mech. 21, 111 (1986).

[11] L. Takác, Acta Math. Hung. 5, 201 (1954). 